\title{
Global Behavior for a Diffusive Predator-Prey Model with Stage Structure and Nonlinear Density Restriction-I: The Case in $\mathbb{R}^{n}$
}

\author{
Rui Zhang, ${ }^{1}$ Ling Guo, ${ }^{2}$ and Shengmao Fu${ }^{2}$ \\ ${ }^{1}$ Department of Mathematics, Lanzhou Jiaotong University, Lanzhou 730070, China \\ ${ }^{2}$ Department of Mathematics, Northwest Normal University, Lanzhou 730070, China \\ Correspondence should be addressed to Shengmao Fu, fusm@nwnu.edu.cn
}

Received 2 April 2009; Accepted 31 August 2009

Recommended by Wenming Zou

This paper deals with a Holling type III diffusive predator-prey model with stage structure and nonlinear density restriction in the space $\mathbb{R}^{n}$. We first consider the asymptotical stability of equilibrium points for the model of ODE type. Then, the existence and uniform boundedness of global solutions and stability of the equilibrium points for the model of weakly coupled reactiondiffusion type are discussed. Finally, the global existence and the convergence of solutions for the model of cross-diffusion type are investigated when the space dimension is less than 6 .

Copyright (C) 2009 Rui Zhang et al. This is an open access article distributed under the Creative Commons Attribution License, which permits unrestricted use, distribution, and reproduction in any medium, provided the original work is properly cited.

\section{Introduction}

Population models with stage structure have been investigated by many researchers, and various methods and techniques have been used to study the existence and qualitative properties of solutions [1-9]. However, most of the discussions in these works are devoted to either systems of ODE or weakly coupled systems of reaction-diffusion equations. In this paper we investigate the global existence and convergence of solutions for a strongly coupled cross-diffusion predator-prey model with stage structure and nonlinear density restriction. Nonlinear problems of this kind are quite difficult to deal with since the usual idea to apply maximum principle arguments to get priori estimates cannot be used here [10].

Consider the following predator-prey model with stage-structure:

$$
X_{1}^{\prime}=B X_{2}-r_{1} X_{1}-C X_{1}-\eta_{1} X_{1}^{2}-\eta_{2} X_{1}^{3}-\frac{E X_{1}^{2} X_{3}}{1+F X_{1}^{2}}
$$




$$
\begin{aligned}
& X_{2}^{\prime}=C X_{1}-r_{2} X_{2}, \\
& X_{3}^{\prime}=-r_{3} X_{3}-\eta_{3} X_{3}^{2}+A X_{3} \frac{E X_{1}^{2}}{1+F X_{1}^{2}},
\end{aligned}
$$

where $X_{1}(t), X_{2}(t)$ denote the density of the immature and mature population of the prey, respectively, $X_{3}(t)$ is the density of the predator. For the prey, the immature population is nonlinear density restriction. $X_{3}$ is assumed to consume $X_{1}$ with Holling type III functional response $E X_{1}^{2} /\left(1+F X_{1}^{2}\right)$ and contributes to its growth with rate $A E X_{1}^{2} /\left(1+F X_{1}^{2}\right)$. For more details on the backgrounds of this model see references $[11,12]$.

Using the scaling $u=\sqrt{F} X_{1}, v=\left(r_{2} \sqrt{F} / C\right) X_{2}, w=\left(E / r_{2} \sqrt{F}\right) X_{3}, d \tau=r_{2} d t$ and redenoting $\tau$ by $t$, we can reduce the system (1.1) to

$$
\begin{aligned}
u^{\prime} & =\beta v-a u-b u^{2}-c u^{3}-\frac{u^{2} w}{1+u^{2}} \equiv f_{1}, \\
v^{\prime} & =u-v \equiv f_{2}, \\
w^{\prime} & =-k w-\gamma w^{2}+\frac{\alpha u^{2} w}{1+u^{2}} \equiv f_{3},
\end{aligned}
$$

where $\beta=B C / r_{2}^{2}, a=\left(r_{1}+C\right) / r_{2}, b=\eta_{1} / r_{2} \sqrt{F}, c=\eta_{2} / r_{2} F, k=r_{3} / r_{2}, \alpha=A E / r_{2} F, \gamma=$ $\eta_{3} \sqrt{F} / E$.

To take into account the natural tendency of each species to diffuse, we are led to the following PDE system of reaction-diffusion type:

$$
\begin{gathered}
u_{t}-d_{1} \Delta u=\beta v-a u-b u^{2}-c u^{3}-\frac{u^{2} w}{1+u^{2}}, \quad x \in \Omega, t>0, \\
v_{t}-d_{2} \Delta v=u-v, \quad x \in \Omega, t>0, \\
w_{t}-d_{3} \Delta w=-k w-r w^{2}+\frac{\alpha u^{2} w}{1+u^{2}}, \quad x \in \Omega, t>0, \\
\partial_{\eta} u=\partial_{\eta} v=\partial_{\eta} w=0, \quad x \in \partial \Omega, t>0, \\
u(x, 0)=u_{0}(x) \geq 0, \quad v(x, 0)=v_{0}(x) \geq 0, \quad w(x, 0)=w_{0}(x) \geq 0, \quad x \in \Omega,
\end{gathered}
$$

where $\Omega$ is a bounded domain in $\mathbb{R}^{n}$ with smooth boundary $\partial \Omega, \eta$ is the outward unit normal vector on $\partial \Omega$, and $\partial_{\eta}=\partial / \partial \eta$. $u_{0}(x), v_{0}(x), w_{0}(x)$ are nonnegative smooth functions on $\bar{\Omega}$. The diffusion coefficients $d_{i}(i=1,2,3)$ are positive constants. The homogeneous Neumann boundary condition indicates that system (1.3) is self-contained with zero population flux across the boundary. The knowledge for system (1.3) is limited (see [13-17]).

In the recent years there has been considerable interest to investigate the global behavior for models of interacting populations with linear density restriction by taking into 
account the effect of self-as well as cross-diffusion [18-26]. In this paper we are led to the following cross-diffusion system:

$$
\begin{aligned}
u_{t} & =\Delta\left[\left(d_{1}+\alpha_{11} u+\alpha_{13} w\right) u\right]+\beta v-a u-b u^{2}-c u^{3}-\frac{u^{2} w}{1+u^{2}}, \quad x \in \Omega, t>0, \\
v_{t} & =\Delta\left[\left(d_{2}+\alpha_{22} v\right) v\right]+u-v, \quad x \in \Omega, t>0 \\
w_{t} & =\Delta\left[\left(d_{3}+\alpha_{33} w\right) w\right]-k w-\gamma w^{2}+\frac{\alpha u^{2} w}{1+u^{2}}, \quad x \in \Omega, t>0, \\
\frac{\partial u}{\partial v} & =\frac{\partial v}{\partial v}=\frac{\partial w}{\partial v}=0, \quad x \in \partial \Omega, t>0, \\
u(x, 0) & =u_{0}(x) \geq 0, \quad v(x, 0)=v_{0}(x) \geq 0, \quad w(x, 0)=w_{0}(x) \geq 0, \quad x \in \Omega,
\end{aligned}
$$

where $d_{1}, d_{2}, d_{3}$ are the diffusion rates of the three species, respectively. $\alpha_{i i}(i=1,2,3)$ are referred as self-diffusion pressures, and $\alpha_{13}$ is cross-diffusion pressure. The term self-diffusion implies the movement of individuals from a higher to a lower concentration region. Crossdiffusion expresses the population fluxes of one species due to the presence of the other species. The value of the cross-diffusion coefficient may be positive, negative, or zero. The term positive cross-diffusion coefficient denotes the movement of the species in the direction of lower concentration of another species and negative cross-diffusion coefficient denotes that one species tends to diffuse in the direction of higher concentration of another species [27]. For $\alpha_{i j} \neq 0$, problem (1.4) becomes strongly coupled with a full diffusion matrix. As far as the authors are aware, very few results are known for cross-diffusion systems with stagestructure.

The main purpose of this paper is to study the asymptotic behavior of the solutions for the reaction-diffusion system (1.3), the global existence, and the convergence of solutions for the cross-diffusion system (1.4). The paper will be organized as follows. In Section 2 a linear stability analysis of equilibrium points for the ODE system (1.2) is given. In Section 3 the uniform bound of the solution and stability of the equilibrium points to the weakly coupled system (1.3) are proved. Section 4 deals with the existence and the convergence of global solutions for the strongly coupled system (1.4).

\section{Global Stability for System (1.2)}

Let $E_{0}=(0,0,0)$. If $\beta>a$, then (1.2) has semitrivial equilibria $E_{1}\left(m_{0}, m_{0}, 0\right)$, where $m_{0}=$ $\left(\sqrt{b^{2}+4 c(\beta-a)}-b\right) / 2 c$. To discuss the existence of the positive equilibrium point of (1.2), we give the following assumptions:

$$
\alpha>k, \quad \beta>a, \quad \sqrt{\frac{k}{\alpha-k}}<m_{0}, \quad \frac{\beta-a-c}{2}+\frac{b^{2}}{8 c} \leq \frac{b \sqrt{p_{1}}}{24 c}+\frac{24(\beta-a) c^{2}}{3 b^{2}+4 c(\beta-a-c)-b \sqrt{p_{1}}},
$$

where $p_{1}=9 b^{2}+24 c(\beta-a-c) \geq 0$. Let one curve $l_{1}: g_{1}(u)=\left(\left(1+u^{2}\right) / u\right)\left(\beta-a-b u-c u^{2}\right)$, and the other curve $l_{3}: g_{3}(u)=k+\gamma w=\alpha u^{2} /\left(1+u^{2}\right)$. Obviously, $l_{1}$ passes the point $\left(m_{0}, 0\right)$. Noting 
that $(\beta-a-c) u^{2}-2 b u^{3}-3 c u^{4}-\beta+a$ attains its maximum at $u=\left(\sqrt{p_{1}}-3 b\right) / 12 c$, thus when $(\beta-a-c) / 2+b^{2} / 8 c \leq b \sqrt{p_{1}} / 24 c+24(\beta-a) c^{2} /\left(3 b^{2}+4 c(\beta-a-c)-b \sqrt{p_{1}}\right), g_{1}^{\prime}(u)<0\left(0<u<m_{0}\right)$. $l_{3}$ has the asymptote $w=\alpha-k / \gamma$ and passes the point $(\sqrt{k / \alpha-k}, 0)$. In this case, $l_{1}$ and $l_{3}$ have unique intersection $\left(u^{*}, w^{*}\right)$, as shown in Figure 1. $E^{*}=\left(u^{*}, v^{*}, w^{*}\right)$ is the unique positive equilibrium point of (1.2), where $v^{*}=u^{*}, w^{*}=\left(\left(1+u^{* 2}\right) / u^{*}\right)\left(\beta-a-b u^{*}-c u^{* 2}\right)$, $k+\gamma w^{*}=\alpha u^{* 2} /\left(1+u^{* 2}\right)$. In addition, the restriction of the existence of the positive equilibrium can be removed, if $\beta<a+c$.

The Jacobian matrix of the equilibrium $E_{0}$ is

$$
J\left(E_{0}\right)=\left(\begin{array}{ccc}
-a & \beta & 0 \\
1 & -1 & 0 \\
0 & 0 & -k
\end{array}\right) .
$$

The characteristic equation of $J\left(E_{0}\right)$ is $(\lambda+k)\left[\lambda^{2}+(1+a) \lambda+a-\beta\right]=0$. $E_{0}$ is a saddle for $\beta>a$. In addition, the dimensions of the local unstable and stable manifold of $E_{0}$ are 1 and 2, respectively. $E_{0}$ is locally asymptotically stable for $\beta<a$.

The Jacobian matrix of the equilibrium $E_{1}$ is

$$
J\left(E_{1}\right)=\left(\begin{array}{ccc}
a_{11} & \beta & -\frac{m_{0}^{2}}{1+m_{0}^{2}} \\
1 & -1 & 0 \\
0 & 0 & a_{33}
\end{array}\right),
$$

where $a_{11}=-a-2 b m_{0}-3 c m_{0}^{2}, a_{33}=-k+\alpha m_{0}^{2} /\left(1+m_{0}^{2}\right)$. The characteristic equation of $J\left(E_{1}\right)$ is $\lambda^{3}+A_{1} \lambda^{2}+B_{1} \lambda+C_{1}=0$, where

$$
\begin{gathered}
A_{1}=-a_{11}-a_{33}+1, \\
B_{1}=a_{11} a_{33}-a_{33}-\left(a_{11}+\beta\right), \\
C_{1}=a_{33}\left(a_{11}+\beta\right), \\
H_{1}=A_{1} B_{1}-C_{1}=\left(a_{11}+a_{33}\right)\left[a_{33}-a_{11} a_{33}+\left(a_{11}+\beta\right)\right]-a_{33}(1+\beta)-\left(a_{11}+\beta\right) .
\end{gathered}
$$

According to Routh-Hurwitz criterion, $E_{1}$ is locally asymptotically stable for $a_{11}+\beta<0$ and $a_{33}<0$, that is, $m_{0}^{2}(\alpha-k)<k$ and $m_{0}>\left(\sqrt{b^{2}+3 c(\beta-a)}-b\right) / 3 c$.

The Jacobian matrix of the equilibrium $E^{*}$ is

$$
J\left(E^{*}\right)=\left(\begin{array}{ccc}
a_{11} & \beta & a_{13} \\
1 & -1 & 0 \\
a_{31} & 0 & a_{33}
\end{array}\right),
$$




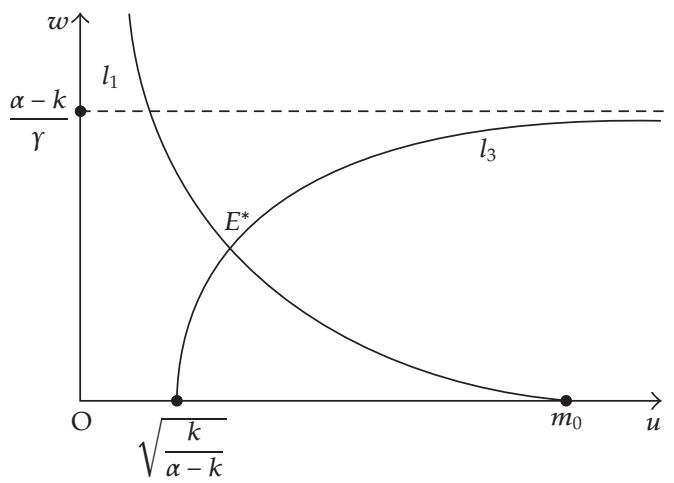

Figure 1

where

$$
\begin{gathered}
a_{11}=-a-2 b u^{*}-3 c u^{* 2}-\frac{2 u^{*} w^{*}}{\left(1+u^{* 2}\right)^{2}}, \quad a_{13}=-\frac{u^{* 2}}{1+u^{* 2}}, \\
a_{31}=\frac{2 \alpha u^{*} w^{*}}{\left(1+u^{* 2}\right)^{2}}, \quad a_{33}=-\gamma w^{*}
\end{gathered}
$$

The characteristic equation of $J\left(E^{*}\right)$ is $\lambda^{3}+A_{2} \lambda^{2}+B_{2} \lambda+C_{2}=0$, where

$$
\begin{gathered}
A_{2}=-a_{11}-a_{33}+1, \\
B_{2}=a_{11} a_{33}-a_{13} a_{31}-a_{33}-\left(a_{11}+\beta\right), \\
C_{2}=a_{33}\left(a_{11}+\beta\right)-a_{13} a_{31}, \\
H_{2}=A_{2} B_{2}-C_{2}=\left(a_{11}+a_{33}\right)\left[a_{13} a_{31}+a_{33}-a_{11} a_{33}+\left(a_{11}+\beta\right)\right]-a_{33}(1+\beta)-\left(a_{11}+\beta\right) .
\end{gathered}
$$

According to Routh-Hurwitz criterion, $E^{*}$ is locally asymptotically stable for $a_{11}+\beta<0$. Obviously, $a_{11}+\beta<0$ can be checked by (2.1).

Now we discuss the global stability of equilibrium points for (1.2).

Theorem 2.1. (i) Assume that (2.1),

$$
\begin{aligned}
b+c u^{*}-\frac{u^{*}\left(\beta-a-b u^{*}\right)}{2+2 \sqrt{1+u^{* 2}}} & >\frac{\left(\sqrt{u^{* 2}+1}+u^{*}\right)^{2}}{8\left(u^{* 2}+1\right)^{2}}+\frac{1}{8}, \\
\frac{\gamma}{\alpha}>\frac{1}{2} &
\end{aligned}
$$

hold, then the equilibrium point $E^{*}$ of (1.2) is globally asymptotically stable. 
(ii) Assume that $\beta>a, m_{0}^{2}(\alpha-k)<k$, and $\left(\sqrt{b^{2}+3 c(\beta-a)}-b\right) / 3 c<m_{0}<2 k / \alpha$ hold, then the equilibrium point $E_{1}$ of (1.2) is globally asymptotically stable. stable.

(iii) Assume that $\beta \leq$ a holds, then the equilibrium point $E_{0}$ of (1.2) is globally asymptotically

Proof. (i) Define the Lyapunov function

$$
\begin{aligned}
E(t)= & \left(u-u^{*}-u^{*} \ln \frac{u}{u^{*}}\right)+\beta\left(v-v^{*}-v^{*} \ln \frac{v}{v^{*}}\right) \\
& +\frac{1}{\alpha}\left(w-w^{*}-w^{*} \ln \frac{w}{w^{*}}\right)
\end{aligned}
$$

Calculating the derivative of $E(t)$ along the positive solution of (1.2), we have

$$
\begin{aligned}
E^{\prime}(t)= & -\frac{\beta}{u^{*}}\left[\sqrt{\frac{v}{u}}\left(u-u^{*}\right)-\sqrt{\frac{u}{v}}\left(v-v^{*}\right)\right]^{2}-\left(u-u^{*}\right)^{2}\left[b+c u+c u^{*}+\frac{w^{*}\left(1-u^{*} u\right)}{\left(1+u^{* 2}\right)\left(1+u^{2}\right)}\right] \\
& -\frac{c}{\alpha}\left(w-w^{*}\right)^{2}+\left(u-u^{*}\right)\left(w-w^{*}\right)\left[\frac{u^{*}+u}{\left(1+u^{* 2}\right)\left(1+u^{2}\right)}-\frac{u}{1+u^{2}}\right] \\
\leq & -\left(u-u^{*}\right)^{2}\left[b+c u+c u^{*}+\frac{w^{*}\left(1-u^{*} u\right)}{\left(1+u^{* 2}\right)\left(1+u^{2}\right)}-\frac{u^{2}}{2\left(1+u^{2}\right)^{2}}-\frac{\left(u+u^{*}\right)^{2}}{2\left(1+u^{* 2}\right)^{2}\left(1+u^{2}\right)^{2}}\right. \\
& \left.+\frac{u\left(u+u^{*}\right)}{\left(1+u^{* 2}\right)\left(1+u^{2}\right)^{2}}\right]-\left(\frac{\gamma}{\alpha}-\frac{1}{2}\right)\left(w-w^{*}\right)^{2} .
\end{aligned}
$$

When $u \in[0, \infty)$, the minimum of $\left(1-u^{*} u\right) /\left(1+u^{2}\right)$ and $u\left(u+u^{*}\right) /\left(1+u^{2}\right)^{2}$ is $-u^{* 2} /(2+$ $\left.2 \sqrt{1+u^{* 2}}\right)$ and 0 , respectively; the maximum of $\left(u+u^{*}\right) /\left(1+u^{2}\right)$ is $u /\left(1+u^{2}\right)$ are $\left(u^{*}+\right.$ $\left.\sqrt{1+u^{* 2}}\right) / 2$ and $1 / 2$, respectively. Thus, when (2.8) hold, $E^{\prime}(t) \leq 0$. According to the Lyapunov-LaSalle invariance principle [28], $E^{*}$ is globally asymptotically stable if (2.1)-(2.3) hold.

(ii) Let

$$
E(t)=\left(u-m_{0}-m_{0} \ln \frac{u}{m_{0}}\right)+\beta\left(v-m_{0}-m_{0} \ln \frac{v}{m_{0}}\right)+\frac{1}{\alpha} w
$$

Then

$$
\begin{aligned}
E^{\prime}(t)= & -\frac{\beta}{m_{0}}\left[\sqrt{\frac{v}{u}}\left(u-m_{0}\right)-\sqrt{\frac{u}{v}}\left(v-m_{0}\right)\right]^{2} \\
& -\left[\left(b+c u+c m_{0}\right)\left(u-m_{0}\right)^{2}+\frac{c}{\alpha} w^{2}-w\left(\frac{m_{0} u}{1+u^{2}}-\frac{k}{\alpha}\right)\right] .
\end{aligned}
$$

Noting that the maximum of $u /\left(1+u^{2}\right)$ is $1 / 2$, and $m_{0}<2 k / \alpha$, we find $m_{0} u /\left(1+u^{2}\right)-k / \alpha<0$. Therefore, $E^{\prime}(t) \leq 0$. 
(iii) Let

$$
E(t)=u+\beta v+\frac{1}{\alpha} w
$$

then

$$
E^{\prime}(t)=(\beta-a) u-b u^{2}-c u^{3}-\frac{k}{\alpha} w-\frac{\gamma}{\alpha} w^{2}
$$

Thus, $E^{\prime}(t) \leq 0$ for $\beta \leq a$. This completes the proof of Theorem 2.1.

\section{Global Behavior of System (1.3)}

In this section we discuss the existence, uniform boundedness of global solutions, and the stability of constant equilibrium solutions for the weakly coupled reaction-diffusion system (1.3). In particular, the unstability results in Section 2 also hold for system (1.3) because solutions of (1.2) are also solutions of (1.3).

Theorem 3.1. Let $u_{0}(x), v_{0}(x), w_{0}(x)$ be nonnegative smooth functions on $\bar{\Omega}$. Then system (1.3) has a unique nonnegative solution $(u(x, t), v(x, t), w(x, t)) \in\left[C(\bar{\Omega} \times[0, \infty)) \cap C^{2,1}(\Omega \times(0, \infty))\right]^{3}$, and

$$
\begin{gathered}
0 \leq u \leq \widehat{M_{1}}=\max \left\{\sup _{\Omega} u_{0}, \sup _{\Omega} v_{0}, \frac{\sqrt{b^{2}+4 c(\beta-a)}-b}{2 c}\right\}, \\
0 \leq v \leq \widehat{M_{2}}=\widehat{M}_{1}, \\
0 \leq w \leq \widehat{M_{3}}=\max \left\{\sup _{\Omega} w_{0}, \frac{\alpha \widehat{M}_{1}^{2}}{r\left(1+\widehat{M}_{1}^{2}\right)}-\frac{k}{r}\right\}
\end{gathered}
$$

on $\bar{\Omega} \times[0, \infty)$. In particular, if $u_{0}, v_{0}, w_{0} \geq(\not \equiv) 0$, then $u, v, w>0$ for all $t>0, x \in \bar{\Omega}$.

Proof. It is easily seen that $\left(f_{1}, f_{2}, f_{3}\right)$ is sufficiently smooth in $\mathbb{R}_{+}^{3}$ and possesses a mixed quasimonotone property in $\mathbb{R}_{+}^{3}$. In addition, $(0,0,0)$ and $\left(\widehat{M_{1}}, \widehat{M_{2}}, \widehat{M_{3}}\right)$ are a pair of lowerupper solutions of problem (1.3) (cf. $\left(\widehat{M_{1}}, \widehat{M_{2}}, \widehat{M_{3}}\right)$ in (3.1)). From [29, Theorem 5.3.4], we conclude that (1.3) exists a unique classical solution $(u, v, w)$ satisfying (3.1). According to strong maximum principle, it follows that $u(x, t), v(x, t), w(x, t)>0, \forall t>0, x \in \bar{\Omega}$. So the proof of the Theorem is completed. 
Remark 3.2. When $c=0$ (namely $\eta_{2}=0$ ), system (1.3) reduces to a system in which the immature population of the prey is linear density restriction. Similar to the proof of Theorem 3.1, we have

$$
\begin{aligned}
& \widehat{M_{1}}=\widehat{M_{2}}=\max \left\{\sup _{\Omega} u_{0}, \sup _{\Omega} v_{0}, \frac{\beta-a}{b}\right\}, \\
& \widehat{M_{3}}=\max \left\{\sup _{\Omega} w_{0}, \frac{\alpha \widehat{M}_{1}^{2}}{r\left(1+\widehat{M}_{1}^{2}\right)}-\frac{k}{r}\right\} .
\end{aligned}
$$

Now we show the local and global stability of constant equilibrium solutions $E_{0}, E_{1}, E^{*}$ for (1.3), respectively.

Theorem 3.3. (i) Assume that (2.1) holds, then the equilibrium point $E^{*}$ of (1.3) is locally asymptotically stable.

(ii) Assume that $\beta>a, m_{0}^{2}(\alpha-k)<k$, and $m_{0}>\sqrt{b^{2}+3 c(\beta-a)}-b / 3 c$ hold, then the equilibrium point $E_{1}$ of (1.3) is locally asymptotically stable. stable.

(iii) Assume that $\beta<a$ holds, then the equilibrium point $E_{0}$ of (1.3) is locally asymptotically

Proof. Let $0=\mu_{1}<\mu_{2}<\mu_{3}<\cdots$ be the eigenvalues of the operator $-\Delta$ on $\Omega$ with Neumann boundary condition, and let $E\left(\mu_{i}\right)$ be the eigenspace corresponding to $\mu_{i}$ in $C^{1}(\bar{\Omega})$. Let

$$
X=\left\{U \in\left[C^{1}(\bar{\Omega})\right]^{3}, \partial_{\eta} U=0, x \in \partial \Omega\right\}, \quad X_{i j}=\left\{c \cdot \phi_{i j}: c \in \mathbb{R}^{3}\right\},
$$

where $\left\{\phi_{i j} ; j=1, \ldots, \operatorname{dim} E\left(\mu_{i}\right)\right\}$ is an orthonormal basis of $E\left(\mu_{i}\right)$, then

$$
X=\oplus_{i=1}^{\infty} X_{i}, \quad X_{i}=\oplus_{j=1}^{\operatorname{dim} E\left(\mu_{i}\right)} X_{i j}
$$

(i) Let $D=\operatorname{diag}\left(d_{1}, d_{2}, d_{3}\right), L=D \Delta+F_{U}\left(E^{*}\right)=D \Delta+\left\{a_{i j}\right\}$, where

$$
\begin{gathered}
a_{11}=-a-2 b u^{*}-3 c u^{* 2}-\frac{2 u^{*} w^{*}}{\left(1+u^{* 2}\right)^{2}}, \quad a_{12}=\beta, \quad a_{13}=-\frac{u^{* 2}}{1+u^{* 2}}, \\
a_{21}=1, \quad a_{22}=-1, \quad a_{23}=0, \\
a_{31}=\frac{2 \alpha u^{*} w^{*}}{\left(1+u^{* 2}\right)^{2}}, \quad a_{32}=0, \quad a_{33}=-\gamma w^{*} .
\end{gathered}
$$


The linearization of (1.3) is $U_{t}=L U$ at $E^{*}$. For each $i \geq 1, X_{i}$ is invariant under the operator $\mathrm{L}$, and $\lambda$ is an eigenvalue of $\mathrm{L}$ on $X_{i}$, if and only if $\lambda$ is an eigenvalue of the matrix $-\mu_{i} D+F_{U}\left(E^{*}\right)$. The characteristic equation is $\varphi_{i}(\lambda)=\lambda^{3}+A_{i} \lambda^{2}+B_{i} \lambda+C_{i}=0$, where

$$
\begin{aligned}
A_{i}= & \mu_{i}\left(d_{1}+d_{2}+d_{3}\right)-a_{11}-a_{33}+1 \\
B_{i}= & \mu_{i}^{2}\left(d_{1} d_{2}+d_{1} d_{3}+d_{2} d_{3}\right) \\
& +\mu_{i}\left[d_{1}\left(1-a_{33}\right)-d_{2}\left(a_{11}+a_{33}\right)+d_{3}\left(1-a_{11}\right)\right] \\
& +a_{11} a_{33}-a_{13} a_{31}-a_{33}-\left(a_{11}+\beta\right) \\
C_{i}= & \mu_{i}^{3} d_{1} d_{2} d_{3}+\mu_{i}^{2}\left(d_{1} d_{3}-a_{33} d_{1} d_{2}-a_{11} d_{2} d_{3}\right) \\
& -\mu_{i}\left[d_{1} a_{33}-d_{2}\left(a_{11} a_{33}-a_{13} a_{31}\right)+d_{3}\left(a_{11}+\beta\right)\right] \\
& +a_{33}\left(a_{11}+\beta\right)-a_{13} a_{31} \\
H_{i}= & A_{i} B_{i}-C_{i}=P_{3} \mu_{i}^{3}+P_{2} \mu_{i}^{2}+P_{1} \mu_{i}+P_{0} \\
P_{3}= & \left(d_{1}+d_{2}\right)\left(d_{1} d_{2}+d_{1} d_{3}+d_{2} d_{3}\right)+d_{3}^{2}\left(d_{1}+d_{2}\right) \\
P_{2}= & \left(d_{1}+d_{2}+d_{3}\right)\left[d_{1}\left(1-a_{33}\right)-d_{2}\left(a_{11}+a_{33}\right)+d_{3}\left(1-a_{11}\right)\right] \\
& -a_{11} d_{1}\left(d_{2}+d_{3}\right)+d_{2}\left(d_{1}+d_{3}\right)-a_{33} d_{3}\left(d_{1}+d_{2}\right), \\
P_{1}= & d_{1}\left[a_{11} a_{33}-a_{13} a_{31}-\left(a_{11}+\beta\right)\right]-d_{2}\left[\left(a_{11}+\beta\right)+a_{33}\right] \\
& +d_{3}\left(a_{11} a_{33}-a_{33}-a_{13} a_{31}\right) \\
& -\left(a_{11}+a_{33}-1\right)\left[d_{1}\left(1-a_{33}\right)-d_{2}\left(a_{11}+a_{33}\right)+d_{3}\left(1-a_{11}\right)\right] \\
P_{0}= & \left(a_{11}+a_{33}\right)\left[a_{13} a_{31}+a_{33}-a_{11} a_{33}+\left(a_{11}+\beta\right)\right] \\
& -a_{33}(1+\beta)-\left(a_{11}+\beta\right) .
\end{aligned}
$$

From Routh-Hurwitz criterion, we can see that three eigenvalues (denoted by $\lambda_{i, 1}, \lambda_{i, 2}, \lambda_{i, 3}$ ) all have negative real parts if and only if $A_{i}>0, C_{i}>0, H_{i}>0$. Noting that $a_{11}, a_{13}, a_{33}<0, a_{31}>$ 0 , we must have $a_{11}+\beta<0$. It is easy to check that $a_{11}+\beta<0$ if $g_{1}^{\prime}\left(u_{1}\right)<0$ (see Section 2).

We can conclude that there exists a positive constant $\delta$, such that

$$
\operatorname{Re}\left\{\lambda_{i, 1}\right\}, \operatorname{Re}\left\{\lambda_{i, 2}\right\}, \operatorname{Re}\left\{\lambda_{i, 3}\right\} \leq-\delta, \quad i \geq 1 .
$$

In fact, let $\lambda=\mu_{i} \xi$, then

$$
\varphi_{i}(\lambda)=\mu_{i}^{3} \xi_{i}^{3}+A_{i} \mu_{i}^{2} \xi_{i}^{2}+B_{i} \mu_{i} \xi+C_{i} \triangleq \widetilde{\varphi}_{i}(\xi)
$$

Since $\mu_{i} \rightarrow \infty$ as $i \rightarrow \infty$, it follows that

$$
\lim _{i \rightarrow \infty} \frac{\tilde{\varphi}_{i}(\xi)}{\mu_{i}^{3}}=\xi^{3}+\left(d_{1}+d_{2}+d_{3}\right) \xi^{2}+\left(d_{1} d_{2}+d_{2} d_{3}+d_{1} d_{3}\right) \xi+d_{1} d_{2} d_{3} \triangleq \tilde{\varphi}(\xi) .
$$


Clearly, $\tilde{\varphi}(\xi)$ has the three roots $-d_{1},-d_{2},-d_{3}$. Let $d=\min \left\{d_{1}, d_{2}, d_{3}\right\}$. By continuity, there exists $i_{0}$ such that the three roots $\xi_{i 1}, \xi_{i 2}, \xi_{i 3}$ of $\tilde{\varphi}_{i}(\xi)=0$ satisfy

$$
\operatorname{Re}\left\{\xi_{i 1}\right\}, \operatorname{Re}\left\{\xi_{i 2}\right\}, \operatorname{Re}\left\{\xi_{i 3}\right\} \leq-\frac{d}{2}, \quad i \geq i_{0}
$$

Let $-\tilde{\delta}=\max _{0 \leq i \leq i_{0}}\left\{\operatorname{Re}\left\{\lambda_{i 1}\right\}, \operatorname{Re}\left\{\lambda_{i 2}\right\}, \operatorname{Re}\left\{\lambda_{i 3}\right\}\right\}$, then $\widetilde{\delta}>0$. Let $\delta=\min \{\tilde{\delta}, d / 2\}$, then (3.7) holds. According to [30, Theorem 5.1.1], we have the locally asymptotically stability of $E^{*}$. and

(ii) The linearization of (1.4) is $U_{t}=L U$ at $E_{1}$, where $L=D \Delta+F_{U}\left(E_{1}\right)=D \Delta+\left\{a_{i j}\right\}$,

$$
\begin{gathered}
a_{11}=-a-2 b m_{0}-3 c m_{0}^{2}, \quad a_{12}=\beta, \quad a_{13}=-\frac{m_{0}^{2}}{1+m_{0}^{2}}, \\
a_{21}=1, \quad a_{22}=-1, \quad a_{23}=0, \\
a_{31}=0, \quad a_{32}=0, \quad a_{33}=-k+\frac{\alpha m_{0}^{2}}{1+m_{0}^{2}} .
\end{gathered}
$$

The characteristic equation of $-\mu_{i} D+F_{U}\left(E_{1}\right)$ is $\varphi_{i}(\lambda)=\lambda^{3}+A_{i} \lambda^{2}+B_{i} \lambda+C_{i}=0$, where

$$
\begin{aligned}
A_{i}= & \mu_{i}\left(d_{1}+d_{2}+d_{3}\right)-a_{11}-a_{33}+1 \\
B_{i}= & \mu_{i}^{2}\left(d_{1} d_{2}+d_{1} d_{3}+d_{2} d_{3}\right) \\
& +\mu_{i}\left[d_{1}\left(1-a_{33}\right)-d_{2}\left(a_{11}+a_{33}\right)+d_{3}\left(1-a_{11}\right)\right] \\
& +a_{11} a_{33}-a_{33}-\left(a_{11}+\beta\right), \\
C_{i}= & \mu_{i}^{3} d_{1} d_{2} d_{3}+\mu_{i}^{2}\left(d_{1} d_{3}-a_{33} d_{1} d_{2}-a_{11} d_{2} d_{3}\right) \\
& -\mu_{i}\left[d_{1} a_{33}-d_{2} a_{11} a_{33}+d_{3}\left(a_{11}+\beta\right)\right]+a_{33}\left(a_{11}+\beta\right), \\
H_{i}= & A_{i} B_{i}-C_{i}=P_{3} \mu_{i}^{3}+P_{2} \mu_{i}^{2}+P_{1} \mu_{i}+P_{0}, \\
P_{3}= & \left(d_{1}+d_{2}\right)\left(d_{1} d_{2}+d_{1} d_{3}+d_{2} d_{3}\right)+d_{3}^{2}\left(d_{1}+d_{2}\right), \\
P_{2}= & \left(d_{1}+d_{2}+d_{3}\right)\left[d_{1}\left(1-a_{33}\right)-d_{2}\left(a_{11}+a_{33}\right)+d_{3}\left(1-a_{11}\right)\right] \\
& -a_{11} d_{1}\left(d_{2}+d_{3}\right)+d_{2}\left(d_{1}+d_{3}\right)-a_{33} d_{3}\left(d_{1}+d_{2}\right), \\
P_{1}= & d_{1}\left[a_{11} a_{33}-\left(a_{11}+\beta\right)\right]-d_{2}\left[\left(a_{11}+\beta\right)+a_{33}\right]+d_{3}\left(a_{11} a_{33}-a_{33}\right) \\
& -\left(a_{11}+a_{33}-1\right)\left[d_{1}\left(1-a_{33}\right)-d_{2}\left(a_{11}+a_{33}\right)+d_{3}\left(1-a_{11}\right)\right], \\
P_{0}= & \left(a_{11}+a_{33}\right)\left[a_{33}-a_{11} a_{33}+\left(a_{11}+\beta\right)\right]-a_{33}(1+\beta)-\left(a_{11}+\beta\right) .
\end{aligned}
$$

The three roots of $\varphi_{i}(\lambda)=0$ all have negative real parts for $a_{11}+\beta<0$ and $a_{33}<0$. Namely, $E_{1}$ is the locally asymptotically stable, if $m_{0}^{2}(\alpha-k)<k$ and $m_{0}>\left(\sqrt{b^{2}+3 c(\beta-a)}-b\right) / 3 c$. 
(iii) The linearization of (1.3) is $U_{t}=L U$ at $E_{0}$, where $L=D \Delta+F_{U}\left(E_{0}\right)=D \Delta+\left\{a_{i j}\right\}$, and

$$
\begin{array}{lrrr}
a_{11}=-a, & a_{12}=\beta, & a_{13}=0, \\
a_{21}=1, & a_{22}=-1, & a_{23}=0, \\
a_{31}=0, & a_{32}=0, & a_{33}=-k .
\end{array}
$$

Similar to (i), $E_{1}$ is locally asymptotically stable, when $\beta<a$.

Remark 3.4. When $c=0$, denote $E_{0}=(0,0,0)$. If $\beta>a$, then (1.3) has the semitrivial equilibrium point $E_{1}=\left(m_{0}, m_{0}, 0\right)$, where $m_{0}=(\beta-a) / b$. If $\alpha>k, \beta>a, k b^{2}<(\alpha-k)(\beta-a)^{2}<$ $27 b^{2}(\alpha-k)$, then (1.3) has a unique positive equilibrium point $E^{*}=\left(u^{*}, v^{*}, w^{*}\right)$. Similar as Theorem 3.3, we have the following.

(i) If $\beta>a, \alpha>k$, and $k b^{2}<(\alpha-k)(\beta-a)^{2}<27 b^{2}(\alpha-k)$ (namely, $\alpha>k, \beta>a$, $\sqrt{k /(\alpha-k)}<(\beta-a) / b<3 \sqrt{3})$, then $E^{*}$ is locally asymptotically stable.

(ii) If $\beta>a$ and $(\alpha-k)(\beta-a)^{2}<k b^{2}$, then $E_{1}$ is locally asymptotically stable.

(iii) If $\beta<a$, then $E_{0}$ is locally asymptotically stable.

Before discussing the global stability, we give an important lemma which has been proved in [31, Lemma 4.1] or in [32, Lemma 2.5.3].

Lemma 3.5. Let $a, b$ be positive constants. Assume that $\phi, \psi \in C^{1}([a, \infty)), \psi(t) \geq 0$, and $\phi$ is bounded from below. If $\phi^{\prime}(t) \leq-b \psi(t)$ and $\psi^{\prime}(t) \leq K(\forall t \geq a)$ for some positive constant $K$, then $\lim _{t \rightarrow \infty} \psi(t)=0$.

Theorem 3.6. (i) Assume that (2.1),

$$
\begin{gathered}
b+c u^{*}-\frac{u^{*}\left(\beta-a-b u^{*}\right)}{2+2 \sqrt{1+u^{* 2}}}>\frac{\left(\sqrt{u^{* 2}+1}+u^{*}\right)^{2}}{8\left(u^{* 2}+1\right)^{2}}+\frac{1}{8}, \\
\frac{\gamma}{\alpha}>\frac{1}{2}
\end{gathered}
$$

hold, then the equilibrium point $E^{*}$ of system (1.3) is globally asymptotically stable.

(ii) Assume that $\beta>a, m_{0}^{2}(\alpha-k)<k$, and $\left(\sqrt{b^{2}+3 c(\beta-a)}-b\right) / 3 c<m_{0}<2 k / \alpha$ hold, then the equilibrium point $E_{1}$ of system (1.3) is globally asymptotically stable.

(iii) Assume that $\beta<a$ and $k>\alpha$ hold, then the equilibrium point $E_{0}$ of system (1.3) is globally asymptotically stable. 
Proof. Let $(u, v, w)$ be the unique positive solution of (1.3). By Theorem 3.1, there exists a positive constant $C$ which is independent of $x \in \bar{\Omega}$ and $t \geq 0$ such that $\|u(\cdot, t)\|_{\infty},\|v(\cdot, t)\|_{\infty}$, $\|w(\cdot, t)\|_{\infty} \leq C$, for $t \geq 0$. By $\left[33\right.$, Theorem $\left.A_{2}\right]$,

$$
\|u(\cdot, t)\|_{C^{2+\alpha}(\bar{\Omega})},\|v(\cdot, t)\|_{C^{2+\alpha}(\bar{\Omega})},\|w(\cdot, t)\|_{C^{2+\alpha}(\bar{\Omega})} \leq C, \quad \forall t \geq t_{0}, \forall t_{0}>0
$$

(i) Define the Lyapunov function

$$
\begin{aligned}
E(t)= & \int_{\Omega}\left(u-u^{*}-u^{*} \ln \frac{u}{u^{*}}\right) \mathrm{d} x+\beta \int_{\Omega}\left(v-v^{*}-v^{*} \ln \frac{v}{v^{*}}\right) \mathrm{d} x \\
& +\frac{1}{\alpha} \int_{\Omega}\left(w-w^{*}-w^{*} \ln \frac{w}{w^{*}}\right) \mathrm{d} x .
\end{aligned}
$$

By Theorem 3.1, $E(t)(t>0)$ is defined well for all solutions of (1.3) with the initial functions $u_{0}, v_{0}, w_{0} \geq(\not \equiv) 0$. It is easily see that $E(t) \geq 0$ and $E(t)=0$ if and only if $u=u^{*}$.

Calculating the derivative of $E(t)$ along positive solution of (1.3) by integration by parts and the Cauchy inequality, we have

$$
\begin{aligned}
E^{\prime}(t)= & -\int_{\Omega}\left(\frac{d_{1} u^{*}}{u^{2}}|\nabla u|^{2}+\beta \frac{d_{2} v^{*}}{v^{2}}|\nabla v|^{2}+\frac{d_{3} w^{*}}{\alpha w^{2}}|\nabla w|^{2}\right) \mathrm{d} x \\
& +\int_{\Omega}\left[\left(u-u^{*}\right) \frac{f_{1}(u, v, w)}{u}+\beta\left(v-v^{*}\right) \frac{f_{2}(u, v, w)}{v}+\frac{1}{\alpha}\left(w-w^{*}\right) \frac{f_{3}(u, v, w)}{w}\right] \mathrm{d} x \\
\leq & -\int_{\Omega}\left(u-u^{*}\right)^{2}\left[b+c u+c u^{*}+\frac{w^{*}\left(1-u^{*} u\right)}{\left(1+u^{* 2}\right)\left(1+u^{2}\right)}-\frac{u^{2}}{2\left(1+u^{2}\right)^{2}}-\frac{\left(u+u^{*}\right)^{2}}{2\left(1+u^{* 2}\right)^{2}\left(1+u^{2}\right)^{2}}\right. \\
& \left.+\frac{u\left(u+u^{*}\right)}{\left(1+u^{* 2}\right)\left(1+u^{2}\right)^{2}}\right] \mathrm{d} x-\left(\frac{\gamma}{\alpha}-\frac{1}{2}\right) \int_{\Omega}\left(w-w^{*}\right)^{2} \mathrm{~d} x .
\end{aligned}
$$

It is not hard to verify that

$$
E^{\prime}(t) \leq-l_{1} \int_{\Omega}\left(u-u^{*}\right)^{2} \mathrm{~d} x-l_{3} \int_{\Omega}\left(w-w^{*}\right)^{2} \mathrm{~d} x
$$

if (3.14) hold. Applying Lemma 3.5, we can obtain

$$
\lim _{t \rightarrow \infty} \int_{\Omega}\left(u-u^{*}\right)^{2} \mathrm{~d} x=0, \quad \lim _{t \rightarrow \infty} \int_{\Omega}\left(w-w^{*}\right)^{2} \mathrm{~d} x=0
$$


Recomputing $E^{\prime}(t)$, we find

$$
\begin{aligned}
E^{\prime}(t) & \leq-\int_{\Omega}\left(\frac{d_{1} u^{*}}{u^{2}}|\nabla u|^{2}+\beta \frac{d_{2} v^{*}}{v^{2}}|\nabla v|^{2}+\frac{d_{3} w^{*}}{\alpha w^{2}}|\nabla w|^{2}\right) \mathrm{d} x \\
& \leq-C \int_{\Omega}\left(|\nabla u|^{2}+|\nabla v|^{2}+|\nabla w|^{2}\right) \mathrm{d} x \triangleq-g(t) .
\end{aligned}
$$

From (3.15), we can see that $g^{\prime}(t)$ is bounded in $\left[t_{0}, \infty\right), t_{0}>0$. It follows from Lemma 3.5 and (3.15) that $g(t) \rightarrow 0$ as $t \rightarrow \infty$. Namely,

$$
\lim _{t \rightarrow \infty} \int_{\Omega}\left(|\nabla u|^{2}+|\nabla v|^{2}+|\nabla w|^{2}\right) \mathrm{d} x=0
$$

Using the Pioncaré inequality, we have

$$
\lim _{t \rightarrow \infty} \int_{\Omega}(u-\bar{u})^{2} \mathrm{~d} x=\lim _{t \rightarrow \infty} \int_{\Omega}(v-\bar{v})^{2} \mathrm{~d} x=\lim _{t \rightarrow \infty} \int_{\Omega}(w-\bar{w})^{2} \mathrm{~d} x=0,
$$

where $\bar{u}(t)=(1 /|\Omega|) \int_{\Omega} u \mathrm{~d} x, \bar{v}(t)=(1 /|\Omega|) \int_{\Omega} v \mathrm{~d} x, \bar{w}(t)=(1 /|\Omega|) \int_{\Omega} w \mathrm{~d} x$. Noting that

$$
\begin{aligned}
|\Omega|\left|\bar{u}(t)-u^{*}\right|^{2} & =\int_{\Omega}\left(\bar{u}-u^{*}\right)^{2} \mathrm{~d} x \leq 2 \int_{\Omega}(\bar{u}-u)^{2} \mathrm{~d} x+2 \int_{\Omega}\left(u-u^{*}\right)^{2} \mathrm{~d} x, \\
|\Omega|\left|\bar{w}(t)-w^{*}\right|^{2} & =\int_{\Omega}\left(\bar{w}-w^{*}\right)^{2} \mathrm{~d} x \leq 2 \int_{\Omega}(\bar{w}-w)^{2} \mathrm{~d} x+2 \int_{\Omega}\left(w-w^{*}\right)^{2} \mathrm{~d} x,
\end{aligned}
$$

according to (3.19) and (3.22), we can see

$$
\bar{u}(t) \rightarrow u^{*}, \quad \bar{w}(t) \rightarrow w^{*} \quad(t \rightarrow \infty)
$$

Thus, there exists $\left\{t_{m}\right\}, \bar{u}^{\prime}\left(t_{m}\right) \rightarrow 0$ as $t_{m} \rightarrow \infty$. Applying the boundness of $\left\{\bar{v}\left(t_{m}\right)\right\}$, there exists a subsequence of $\left\{\bar{v}\left(t_{m}\right)\right\}$, denoted still by $\left\{\bar{v}\left(t_{m}\right)\right\}$, such that $\bar{v}\left(t_{m}\right) \rightarrow \widehat{v}$. On the one hand

$$
\left.\int_{\Omega} u_{t} \mathrm{~d} x\right|_{t_{m}}=|\Omega| \bar{u}^{\prime}\left(t_{m}\right) \longrightarrow 0, \quad t_{m} \longrightarrow \infty
$$

On the other hand

$$
\begin{aligned}
\left.\int_{\Omega} u_{t} \mathrm{~d} x\right|_{t_{m}} & =\left.\int_{\Omega}\left(d_{1} \Delta u+f_{1}(u, v, w)\right) \mathrm{d} x\right|_{t_{m}}=\left.\int_{\Omega} f_{1}(u, v, w) \mathrm{d} x\right|_{t_{m}} \\
& =\left.\int_{\Omega}\left[\beta\left(v-v^{*}\right)-\left(a+b\left(u+u^{*}\right)+c\left(u^{2}+u u^{*}+u^{* 2}\right)\right)\left(u-u^{*}\right)-d u\left(w-w^{*}\right)\right] \mathrm{d} x\right|_{t_{m}} .
\end{aligned}
$$


According to (3.19) to compute the limit of the previous equation and using the uniqueness of the limit, we have $\widehat{v}=v^{*}$, and

$$
\lim _{t_{m} \rightarrow \infty} \bar{v}\left(t_{m}\right)=v^{*}
$$

It follows from (3.15) that there exists a subsequence of $\left\{t_{m}\right\}$, denoted still by $\left\{t_{m}\right\}$, and nonnegative functions $g_{i} \in C^{2}(\bar{\Omega}), i=1,2,3$, such that

$$
u\left(\cdot, t_{m}\right) \longrightarrow g_{1}(\cdot), \quad v\left(\cdot, t_{m}\right) \longrightarrow g_{2}(\cdot), \quad w\left(\cdot, t_{m}\right) \longrightarrow g_{3}(\cdot) \quad \text { in } C^{2}(\bar{\Omega})
$$

Applying (3.19)-(3.27), we obtain that $g_{1}=u^{*}, g_{2}=v^{*}, g_{3}=w^{*}$, and

$$
u\left(\cdot, t_{m}\right) \longrightarrow u^{*}, \quad v\left(\cdot, t_{m}\right) \longrightarrow v^{*}, \quad w\left(\cdot, t_{m}\right) \longrightarrow w^{*} \quad \text { in } C^{2}(\bar{\Omega})
$$

In view of Theorem 3.3, we can conclude that $E^{*}$ is globally asymptotically stable.

(ii) Let

$$
E(t)=\int_{\Omega}\left(u-m_{0}-m_{0} \ln \frac{u}{m_{0}}\right) \mathrm{d} x+\beta \int_{\Omega}\left(v-m_{0}-m_{0} \ln \frac{v}{m_{0}}\right) \mathrm{d} x+\frac{1}{\alpha} \int_{\Omega} w \mathrm{~d} x .
$$

Then

$$
\begin{aligned}
E^{\prime}(t)= & -m_{0} \int_{\Omega}\left(\frac{d_{1}}{u^{2}}|\nabla u|^{2}+\beta \frac{d_{2}}{v^{2}}|\nabla v|^{2}\right) \mathrm{d} x \\
& +\int_{\Omega}\left[\left(u-u^{*}\right) \frac{f_{1}(u, v, w)}{u}+\beta\left(v-v^{*}\right) \frac{f_{2}(u, v, w)}{v}+\frac{1}{\alpha} f_{3}(u, v, w)\right] \mathrm{d} x \\
\leq & -\int_{\Omega} \frac{\beta}{m_{0}}\left[\sqrt{\frac{v}{u}}\left(u-m_{0}\right)-\sqrt{\frac{u}{v}}\left(v-m_{0}\right)\right]^{2} \\
& -\int_{\Omega}\left[\left(b+c u+c m_{0}\right)\left(u-m_{0}\right)^{2}+\frac{\gamma}{\alpha} w^{2}-w\left(\frac{m_{0} u}{1+u^{2}}-\frac{k}{\alpha}\right)\right] \mathrm{d} x .
\end{aligned}
$$

Therefore, $E^{\prime}(t) \leq-\left(b+c m_{0}\right) \int_{\Omega}\left(u-m_{0}\right)^{2} \mathrm{~d} x-\frac{\gamma}{\alpha} \int_{\Omega} w^{2} \mathrm{~d} x$. It follows that the equilibrium point $E_{1}$ of (1.3) is globally asymptotically stable.

(iii) Define

$$
E(t)=\frac{1}{2} \int_{\Omega}\left(u^{2}+\beta v^{2}+w^{2}\right) \mathrm{d} x
$$


Then

$$
\begin{aligned}
E^{\prime}(t)= & -\int_{\Omega}\left(d_{1}|\nabla u|^{2}+\beta d_{2}|\nabla v|^{2}+d_{3}|\nabla w|^{2}\right) \mathrm{d} x \\
& +\int_{\Omega}\left(u f_{1}(u, v, w)+\beta v f_{2}(u, v, w)+w f_{3}(u, v, w)\right) \mathrm{d} x .
\end{aligned}
$$

When $a>\beta, k>\alpha$,

$$
E^{\prime}(t) \leq-\int_{\Omega}\left[a u^{2}+\beta v^{2}+(k-\alpha) w^{2}\right] \mathrm{d} x .
$$

The following proof is similar to (i).

Remark 3.7. When $c=0$, Theorem 3.6 shows the following.

(i) Assume that $\beta>a, \alpha>k, \sqrt{k /(\alpha-k)}<(\beta-a) / b<3 \sqrt{3}$,

$$
b-\frac{u^{*}\left(\beta-a-b u^{*}\right)}{2+2 \sqrt{1+u^{* 2}}}>\frac{\left(\sqrt{u^{* 2}+1}+u^{* 2}\right)^{2}}{8\left(u^{* 2}+1\right)^{2}}+\frac{1}{8}, \quad \frac{\gamma}{\alpha}>\frac{1}{2}
$$

hold, then the equilibrium point $E^{*}$ of (1.3) is globally asymptotically stable.

(ii) Assume that $\beta>a$ and $b^{2} k /(\beta-a)>\max \{(\alpha-k)(\beta-a), b \alpha / 2\}$ hold, then the equilibrium point $E_{1}$ of (1.3) is globally asymptotically stable.

(iii) Assume that $\beta<a$ and $k>\alpha$ hold, then the equilibrium point $E_{0}$ of (1.3) is globally asymptotically stable.

Example 3.8. Consider the following system:

$$
\begin{aligned}
X_{1 t}-D_{1} \Delta X_{1} & =5 X_{2}-0.6 X_{1}-1.4 X_{1}-2 X_{1}^{2}-6 X_{1}^{3}-X_{3} \frac{2 X_{1}^{2}}{1+2 X_{1}^{2}}, \quad x \in \Omega, t>0, \\
X_{2 t}-D_{2} \Delta X_{2} & =1.4 X_{1}-X_{2}, \quad x \in \Omega, t>0 \\
X_{3 t}-D_{3} \Delta X_{3} & =-X_{3}-\sqrt{2} X_{3}^{2}+X_{3} \frac{2 X_{1}^{2}}{1+2 X_{1}^{2}}, \quad x \in \Omega, t>0 \\
\partial_{\eta} X_{i} & =0, \quad i=1,2,3, x \in \partial \Omega, t>0 \\
X_{i}(x, 0) & =X_{i 0}(x) \geq 0, \quad i=1,2,3, x \in \Omega .
\end{aligned}
$$

Using the software Matlab, one can obtain $u^{*}=v^{*}=1.1274, w^{*}=0.1199$. It is easy to see that the previous system satisfies the all conditions of Theorem 3.6(i). So the positive equilibrium point $(0.5637,0.5637,0.1199)$ of the previous system is globally asymptotically stable. 


\section{Global Existence and Stability of Solutions for the System (1.4)}

By [34-36], we have the following result.

Theorem 4.1. If $u_{0}, v_{0}, w_{0} \in W_{p}^{1}(\Omega), p>n$, then (1.4) has a unique nonnegative solution $u, v, w \in$ $C\left([0, T), W_{p}^{1}(\Omega)\right) \cap C^{\infty}\left((0, T), C^{\infty}(\Omega)\right)$, where $T \leq+\infty$ is the maximal existence time of the solution. If the solution $(u, v, w)$ satisfies the estimate

$$
\sup \left\{\|u(\cdot, t)\| W_{p}^{1}(\Omega),\|v(\cdot, t)\| W_{p}^{1}(\Omega),\|w(\cdot, t)\| W_{p}^{1}(\Omega): 0<t<T\right\}<\infty,
$$

then $T=+\infty$. If, in addition, $u_{0}, v_{0}, w_{0} \in W_{p}^{2}(\Omega)$, then $u, v, w \in C\left([0, \infty), W_{p}^{2}(\Omega)\right)$.

In this section, we consider the existence and the convergence of global solutions to the system (1.4).

Theorem 4.2. Let $\alpha_{11}, \alpha_{22}>0$ and the space dimension $n<6$. Suppose that $u_{0}, v_{0}, w_{0} \in$ $C^{2+\lambda}(\bar{\Omega})(0<\lambda<1)$ are nonnegative functions and satisfy zero Neumann boundary conditions. Then (1.4) has a unique nonnegative solution $u, v, w \in C^{2+\lambda, 1+\lambda / 2}(\bar{\Omega} \times[0, \infty))$.

In order to prove Theorem 4.2, some preparations are collected firstly.

Lemma 4.3. Let $(u, v, w)$ be a solution of (1.4). Then

$$
\begin{gathered}
u, v \geq 0, \quad 0 \leq w \leq M_{1}, \quad \text { in } Q_{T} \equiv \Omega \times(0, T), \\
\sup _{0<t<T}\|u(\cdot, t)\|_{L^{1}(\Omega)}, \sup _{0<t<T}\|v(\cdot, t)\|_{L^{1}(\Omega)} \leq C_{1}(T), \\
\|u\|_{L^{2}\left(Q_{T}\right)},\|v\|_{L^{2}\left(Q_{T}\right)} \leq C_{2}(T),
\end{gathered}
$$

where $M_{1}=\max \left\{\alpha / \gamma,\left\|w_{0}\right\|_{L^{\infty}(\Omega)}\right\}$.

Proof. From the maximum principle for parabolic equations, it is not hard to verify that $u, v, w \geq 0$ and $w$ is bounded.

Multiplying the second equation of (1.4) by $(a+\beta)$, adding up the first equation of (1.4), and integrating the result over $\Omega$, we obtain

$$
\frac{d}{d t} \int_{\Omega}[u+(a+\beta) v] d x \leq-a \int_{\Omega} v d x+\int_{\Omega}\left(\beta u-b u^{2}\right) d x .
$$

Using Young inequality and Hölder inequality, we have

$$
\int_{\Omega}\left(\beta u-b u^{2}\right) d x \leq C_{2,1}-\frac{a}{a+\beta} \int_{\Omega} u d x
$$


where $C_{2,1}=(1 / 4 b)(\beta+a /(a+\beta))^{2}|\Omega|$. It follows from (4.3) and (4.4) that

$$
\frac{d}{d t} \int_{\Omega}[u+(a+\beta) v] d x \leq C_{2,1}-\frac{a}{a+\beta} \int_{\Omega}[u+(a+\beta) v] d x .
$$

Thus,

$$
\|u(\cdot, t)\|_{L^{1}(\Omega)},\|v(\cdot, t)\|_{L^{1}(\Omega)} \leq C_{2,2}
$$

where $C_{2,2}$ depends on $\left\|v_{0}\right\|_{L^{1}(\Omega)},\left\|u_{0}\right\|_{L^{1}(\Omega)}$ and coefficients of (1.4). In addition, there exists a positive constant $C_{1}(T)$, such that

$$
\sup _{0<t<T}\|u(\cdot, t)\|_{L^{1}(\Omega)} \sup _{0<t<T}\|v(\cdot, t)\|_{L^{1}(\Omega)} \leq C_{1}(T) .
$$

Integrating the first equation of (1.4) over $\Omega$, we have

$$
\frac{d}{d t} \int_{\Omega} u d x \leq \beta \int_{\Omega} v d x-b \int_{\Omega} u^{2} d x
$$

Integrating (4.8) from 0 to $T$, we have

$$
\int_{\Omega} u(x, T) d x-\int_{\Omega} u(x, 0) d x \leq \beta \int_{0}^{T} \int_{\Omega} v d x d t-b \int_{0}^{T} \int_{\Omega} u^{2} d x d t .
$$

According to (4.7), there exists a positive constant $C_{2}(T)$, such that

$$
\|u\|_{L^{2}\left(Q_{T}\right)} \leq C_{2}(T) .
$$

Multiplying the second equation of (1.4) by $v$ and integrating it over $\Omega$, we obtain

$$
\begin{aligned}
\frac{1}{2} \frac{d}{d t} \int_{\Omega} v^{2} d x & =-\int_{\Omega}\left(d_{2}+2 \alpha_{22} v\right)|\nabla v|^{2} d x+\int_{\Omega}\left(u v-v^{2}\right) d x \\
& \leq \frac{1}{2} \int_{\Omega} u^{2} d x-\frac{1}{2} \int_{\Omega} v^{2} d x
\end{aligned}
$$

Integrating the previous inequation from 0 to $T$, we have

$$
\|v\|_{L^{2}\left(Q_{T}\right)} \leq C_{2}(T) .
$$

Lemma 4.4. Let $(u, v, w)$ be a solution of $(1.4), w_{1}=\left(d_{3}+\alpha_{33} w\right) w$, and $\tau<T$. Then there exists $a$ positive constant $C_{3}(\tau)$ depending on $\left\|w_{0}\right\|_{W_{2}^{1}(\Omega)}$ and $\left\|w_{0}\right\|_{L^{\infty}(\Omega)}$, such that

$$
\left\|w_{1}\right\|_{W_{2}^{2,1}\left(Q_{\tau}\right)} \leq C_{3}(\tau)
$$

Furthermore $\nabla w_{1} \in V_{2}\left(Q_{\tau}\right)$ and $\nabla w_{1} \in L^{2(n+2) / n}\left(Q_{\tau}\right)$. 
Proof. $w_{1}$ satisfies the equation

$$
w_{1 t}=\left(d_{3}+2 \alpha_{33} w\right) \Delta w_{1}+c_{1}+c_{2} \frac{u^{2}}{1+u^{2}}
$$

where $c_{1}, c_{2}$ are functions of $w$ and so are bounded because of Lemma 4.3.

Multiply the second equation of (1.4) by $-\Delta w_{1}$ and integrate it over $Q_{\tau}$ to obtain

$$
\begin{aligned}
& \frac{1}{2} \int_{\Omega}\left|\nabla w_{1}\right|^{2}(x, \tau) d x-\frac{1}{2} \int_{\Omega}\left|\nabla w_{1}\right|^{2}(x, 0) d x+d_{3} \int_{Q_{\tau}}\left|\nabla w_{1}\right|^{2} d x d s \\
& \quad \leq \int_{Q_{\tau}}\left|\Delta w_{1}\right|\left|c_{1}+c_{2} \frac{u^{2}}{1+u^{2}}\right| d x d s \\
& \quad \leq m_{1}\left\|\Delta w_{1}\right\|_{L^{2}\left(Q_{\tau}\right)} \\
& \quad \leq \frac{d_{3}}{2}\left\|\Delta w_{1}\right\|_{L^{2}\left(Q_{\tau}\right)}^{2}+\frac{m_{1}}{2 d_{3}} .
\end{aligned}
$$

Then

$$
\begin{gathered}
\int_{\Omega}\left|\nabla w_{1}\right|^{2}(x, \tau) d x+d_{3} \int_{Q_{\tau}}\left|\nabla w_{1}\right|^{2} d x d s \\
\leq \int_{\Omega}\left|\nabla w_{1}\right|^{2}(x, 0) d x+\frac{m_{1}}{2 d_{3}},
\end{gathered}
$$

and $w_{1} \in W_{2}^{2,1}\left(Q_{T}\right)$. From a disposal similar to the proof of Lemma 2.2 in [23], we have $\nabla w_{1} \in V_{2}\left(Q_{\tau}\right)$. Using a standard embedding result, we obtain $\nabla w_{1} \in L^{2(n+2) / n}\left(Q_{\tau}\right)$.

Lemma 4.5 (see [23, Lemmas 2.3 and 2.4]). Let $q>1, \tilde{q}=2+4 q / n(q+1), \tilde{\beta} \in(0,1)$, and let $C_{T}>0$ be any number which may depend on $T$. Then there is a constant $M_{2}$ depending on $n, q, \Omega, \tilde{\beta}$, and $C_{T}$ such that

$$
\|g\|_{L_{\left(Q_{T}\right)}^{\tilde{q}}} \leq M_{2}\left\{1+\left(\sup _{0 \leq t \leq T}\|g(\cdot, t)\|_{L^{2 q /(q+1)}(\Omega)}\right)^{4 q / n(q+1) \tilde{q}}\|\nabla g\|_{L^{2}\left(Q_{T}\right)}^{2 / \tilde{q}}\right\},
$$

for any $g \in C\left([0, T), W_{2}^{1}(\Omega)\right)$ with $\left(\int_{\Omega}|g(\cdot, t)|^{\tilde{\beta}} d x\right)^{1 / \tilde{\beta}} \leq C_{T}$ for all $t \in[0, T]$. lemma.

To obtain $L^{\infty}$-estimates of $u, v$, we establish $L^{q}$-estimates of $u, v$ in the following

Lemma 4.6. Let $\alpha_{11}, \alpha_{22}>0,1<q<2(n+1) /(n-2)$, then there exist positive constants $C(q, T)$ and $C(T)$, such that

$$
\|u\|_{L^{q}\left(Q_{T}\right)},\|v\|_{L^{q}\left(Q_{T}\right)} \leq C(q, T), \quad\|u\|_{V_{2}\left(Q_{T}\right)},\|v\|_{V_{2}\left(Q_{T}\right)} \leq C(T) .
$$


Proof. Multiply the first equation of (1.4) by $q \mathcal{u}^{q-1}$ for $q>1$ and integrate by parts over $\Omega$ to obtain

$$
\begin{aligned}
\frac{d}{d t} \int_{\Omega} u^{q} d x \leq & -q(q-1) \int_{\Omega} u^{q-2}\left(d_{1}+2 \alpha_{11} u\right)|\nabla u|^{2} d x \\
& -\alpha_{13}(q-1) \int_{\Omega} \nabla\left(u^{q}\right) \cdot \nabla w d x+q \beta \int_{\Omega} u^{q-1} v d x
\end{aligned}
$$

Integrating (4.19) from 0 to $t$, we have

$$
\begin{gathered}
\int_{\Omega} u^{q}(x, t) d x-\int_{\Omega} u_{0}^{q}(x) d x+q(q-1) \int_{Q_{t}} u^{q-2}\left(d_{1}+2 \alpha_{11} u\right)|\nabla u|^{2} d x d s \\
\quad \leq-\alpha_{13}(q-1) \int_{Q_{t}} \nabla\left(u^{q}\right) \cdot \nabla w d x d s+q \beta \int_{Q_{t}} u^{q-1} v d x d s .
\end{gathered}
$$

Then substitution of $u^{q-2}|\nabla u|^{2}=\left(4 / q^{2}\right)\left|\nabla\left(u^{q / 2}\right)\right|^{2}, u^{q-1}|\nabla u|^{2}=\left(4 /(q+1)^{2}\right)\left|\nabla\left(u^{(q+1) / 2}\right)\right|^{2}$ into (4.20) leads to

$$
\begin{aligned}
& \int_{\Omega} u^{q}(x, t) d x+\frac{4(q-1) d_{1}}{q} \int_{Q_{t}}\left|\nabla\left(u^{q / 2}\right)\right|^{2} d x d s+\frac{8 \alpha_{11} q(q-1)}{(q+1)^{2}} \int_{Q_{t}}|\nabla(u(q+1) / 2)|^{2} d x d s \\
& \leq \int_{\Omega} u_{0}^{q}(x) d x-\alpha_{13}(q-1) \int_{Q_{t}} \nabla\left(u^{q}\right) \cdot \nabla w d x d s+q \beta \int_{Q_{t}} u^{q-1} v .
\end{aligned}
$$

It follows from Hölder inequality and Lemma 4.3 that

$$
\begin{aligned}
q \beta \int_{Q_{t}} u^{q-1} v & \leq q \beta\left\|u^{(q-1) / 2}\right\|_{L^{n+2}\left(Q_{T}\right)}\left\|u^{(q-1) / 2}\right\|_{L^{2(n+2) / n}\left(Q_{T}\right)}\|v\|_{L^{2}\left(Q_{T}\right)} \\
& \leq C_{3,1}\|u\|_{L^{(q-1)(n+2) / 2}\left(Q_{T}\right)}^{q-1} .
\end{aligned}
$$

Note that $1 / 2+1 /(n+2)+n / 2(n+2)=1$, and $n+2 \geq 2(n+2) / n$ for $n \geq 2$. From Hölder inequality, Young inequality, and Lemma 4.4, we have

$$
\begin{aligned}
\left|\int_{Q_{T}} \nabla\left(u^{q}\right) \cdot \nabla w d x d t\right| & =\frac{2 q}{q+1}\left|\int_{Q_{T}} u^{(q-1) / 2} \nabla w \cdot \nabla\left(u^{(q+1) / 2}\right) d x d t\right| \\
& \leq \frac{2 q}{q+1}\|u\|_{L^{(q-1)(n+2) / 2}\left(Q_{T}\right)}^{(q-1) / 2}\|\nabla w\|_{L^{2(n+2) / n}\left(Q_{T}\right)}\left\|\nabla\left(u^{(q+1) / 2}\right)\right\|_{L^{2}\left(Q_{T}\right)} \\
& \leq C_{3,2}\|u\|_{L^{(q-1)(n+2) / 2}\left(Q_{T}\right)}^{(q-1) / 2}\left\|\nabla\left(u^{(q+1) / 2}\right)\right\|_{L^{2}\left(Q_{T}\right)} \\
& \leq \frac{C_{3,2} \epsilon_{1}}{2}\left\|\nabla\left(u^{(q+1) / 2}\right)\right\|_{L^{2}\left(Q_{T}\right)}^{2}+\frac{C_{3,2}}{2 \epsilon_{1}}\|u\|_{L^{(q-1)(n+2) / 2}\left(Q_{T}\right)}^{q-1} .
\end{aligned}
$$


Substitution of (4.22) and (4.23) into (4.21) leads to

$$
\begin{gathered}
\int_{\Omega} u_{1}^{2 q /(q+1)}(x, t) d x+\frac{4(q-1) d_{1}}{q} \int_{Q_{t}}\left|\nabla\left(u^{q / 2}\right)\right|^{2} d x d t+\frac{8 \alpha_{11} q(q-1)}{(q+1)^{2}} \int_{Q_{t}}\left|\nabla u_{1}\right|^{2} d x d t \\
\leq \int_{\Omega} u_{0}^{q}(x) d x+\epsilon C_{3,3}\left\|\nabla u_{1}\right\|_{L^{2}\left(Q_{T}\right)}^{2}+\frac{C_{3,4}}{\epsilon}\left\|u_{1}\right\|_{L^{(q-1)(n+2) /(q+1)}\left(Q_{T}\right)^{\prime}}^{2(q-1) /(q+1)}
\end{gathered}
$$

where $\epsilon>0$ is arbitrary and $u_{1}=u^{(q+1) / 2}$.

Choose $\epsilon$ such that

$$
\epsilon \alpha_{13} C_{3,3}<\frac{4 \alpha_{11} q}{(q+1)^{2}}
$$

then it follows from (4.24) that

$$
\sup _{0<t<T} \int_{\Omega} u_{1}^{2 q /(q+1)}(x, t) d x+\int_{Q_{T}}\left|\nabla u_{1}\right|^{2} d x d t \leq C_{3,5}\left(1+\left\|u_{1}\right\|_{L^{(q-1)(n+2) /(q+1)}\left(Q_{T}\right)}^{2(q-1) /(q+1)}\right) .
$$

Let

$$
E \equiv \sup _{0<t<T} \int_{\Omega} u_{1}^{2 q /(q+1)}(x, t) d x+\int_{Q_{T}}\left|\nabla u_{1}\right|^{2} d x d t
$$

Then $(q-1)(n+2) /(q+1)<\tilde{q}$ for

$$
1<q<\frac{n(n+4)}{n^{2}-4}
$$

According to Lemma 4.5 and the definition of $E$, we can see

$$
\left\|u_{1}\right\|_{L^{\tilde{q}}\left(Q_{T}\right)} \leq M_{3}\left(1+E^{2 / n \tilde{q}} E^{1 / \tilde{q}}\right) .
$$

Combining (4.26) and (4.29), we have

$$
\begin{aligned}
E & \leq C_{3,5}\left(1+\left\|u_{1}\right\|_{L^{\tilde{q}}\left(Q_{T}\right)}^{2(q-1) /(q+1)}\right) \\
& \leq C_{3,5}\left\{1+\left[M_{3}\left(1+E^{2 / n \tilde{q}} E^{1 / \tilde{q}}\right)\right]^{2(q-1) /(q+1)}\right\} \\
& \leq C_{3,6}\left(1+E^{\mu}\right),
\end{aligned}
$$

where $\mu=(2(q-1) / \widetilde{q}(q+1))(2 / n+1)<1 / \widetilde{q}[4 q / n(q+1)+2]=1$. Therefore $E$ is bounded from (4.30). 
From (4.29), we have $u_{1} \in L^{\tilde{q}}\left(Q_{T}\right)$. Namely, $u \in L^{r}\left(Q_{T}\right), r=\tilde{q}(q+1) / 2=q+1+2 q / n$. Combining (4.28), we have $u \in L^{r}\left(Q_{T}\right)$, where $r<2(n+1) /(n-2)$.

Setting $q=2$ in (4.20) (it is easily checked that $q=2<n(n+4) /\left(n^{2}-4\right)$, i.e., $\left.n=2,3,4,5\right)$, we have $\|u\|_{V_{2}\left(Q_{T}\right)} \leq C_{T}$.

Multiplying the second equation of (1.4) by $q v^{q-1}$ and integrating it over $\Omega$, we have

$$
\frac{d}{d t} \int_{\Omega} v^{q} d x=-q(q-1) \int_{\Omega} v^{q-2}\left(d_{2}+2 \alpha_{22} v\right)|\nabla v|^{2} d x+q \int_{\Omega} v^{q-1}(u-v) d x
$$

The result of $v$ can be obtained from an analogue of the previous proof of $u$ 's.

Lemma 4.7. Let $n=2,3,4,5$, then there exists a positive constant $M_{4}$ such that

$$
\|u\|_{L^{\infty}\left(Q_{T}\right)},\|v\|_{L^{\infty}\left(Q_{T}\right)} \leq M_{4} .
$$

Proof. We will prove this lemma by [37, Theorem 7.1, page 181]. At first, we rewrite the first two equations of (1.4) as

$$
\begin{gathered}
\frac{\partial u}{\partial t}-\sum_{i, j=1}^{n} \frac{\partial}{\partial x_{i}}\left(a_{i j} \frac{\partial u}{\partial x_{j}}\right)-\sum_{i=1}^{n} \frac{\partial}{\partial x_{i}}\left(a_{i} u\right)+u\left(a+b u+c u^{2}+\frac{u w}{1+u^{2}}\right)=\beta v \\
\frac{\partial v}{\partial t}-\sum_{i, j=1}^{n} \frac{\partial}{\partial x_{i}}\left(b_{i j} \frac{\partial v}{\partial x_{j}}\right)+v=u,
\end{gathered}
$$

where $a_{i j}(x, t)=\left(d_{1}+2 \alpha_{11} u+\alpha_{13} w\right) \delta_{i j}, a_{i}(x, t)=\alpha_{13}\left(\partial w / \partial x_{i}\right), b_{i j}(x, t)=\left(d_{2}+2 \alpha_{22} v\right) \delta_{i j}, \delta_{i j}$ is Kronecker symbol. It follows from Lemma 4.6 that $u \in L^{q}\left(Q_{T}\right), 1<q<2(n+1) /(n-2)$.

By the third equation of (1.4), we have

$$
w_{t}=\nabla\left[\left(d_{3}+2 \alpha_{33} w\right) \nabla w\right]-k w-r w^{2}+\frac{\alpha u^{2} w}{1+u^{2}} .
$$

It follows from Lemma 4.3 that $d_{3}+2 \alpha_{33} w,-k w-\gamma w^{2}+\alpha u^{2} w /\left(1+u^{2}\right)$ is bounded in $Q_{T}$. Applying Theorem 10.1 [37, Page 204] to (4.34), we have

$$
w \in C^{\lambda_{1}, \lambda_{1} / 2}\left(\bar{Q}_{T}\right), \quad \lambda_{1}>0 .
$$

Recall that $w_{1}=\left(d_{3}+\alpha_{33} w\right) w$ satisfy (4.14) in Lemma 4.4 , that is,

$$
w_{1 t}=\left(d_{3}+\alpha_{33} w\right) \Delta w_{1}+c_{1}+c_{2} \frac{u^{2}}{1+u^{2}}
$$

where $c_{1}+c_{2}\left(u^{2} /\left(1+u^{2}\right)\right)$ is bounded. Since $d_{3}+2 \alpha_{33} w \in C^{1_{1}, \lambda_{1} / 2}\left(\bar{Q}_{T}\right)$ by (4.35), applying Theorem 9.1 [37, page 341-342] to (4.36), we have

$$
w_{1} \in W_{q}^{2,1}\left(Q_{T}\right)
$$


It follows from [37, Lemma 3.3, page 80] that $\nabla w_{1} \in L^{(n+2) q /(n+2-q)}\left(Q_{T}\right)$ and so $\nabla w=$ $\nabla w_{1} /\left(d_{3}+2 \alpha_{33} w\right) \in L^{(n+2) q /(n+2-q)}\left(Q_{T}\right)$. Recall from Lemma 4.6 that $u, v \in V_{2}\left(Q_{T}\right)$, so that $u, v \in L^{\infty}\left(Q_{T}\right)$ by applying Theorem 7.1 [37, Page 181] to (4.33).

Proof of Theorem 4.2. Firstly, Theorem 4.2 can be proved in a similar way as Theorem 2 in [21, 25] when the space dimension $n=1$.

Secondly, for $2 \leq n<6$, applying Lemma 3.3 [37, Page 80] to (4.36), we have

$$
w_{1} \in C^{1+\lambda_{2},\left(1+\lambda_{2}\right) / 2}\left(\bar{Q}_{T}\right), \quad 0<\lambda_{2}<1
$$

Since $w=\left(-d_{3}+\sqrt{d_{3}^{2}+4 \alpha_{33} w_{1}}\right) / 2 \alpha_{33}$, we obtain

$$
w \in C^{1+\lambda_{2},\left(1+\lambda_{2}\right) / 2}\left(\bar{Q}_{T}\right), \quad 0<\lambda_{2}<1 .
$$

The first two equations can be written in the divergence form as

$$
\begin{gathered}
u_{t}=\nabla\left[\left(d_{1}+2 \alpha_{11} u+\alpha_{13} w\right) \nabla u+\alpha_{13} u \nabla w\right]+g_{1}(x, t), \\
v_{t}=\nabla\left[\left(d_{2}+2 \alpha_{22} v\right) \nabla v\right]+g_{2}(x, t),
\end{gathered}
$$

where $g_{1}=\beta v-a u-b u^{2}-c u^{3}-u^{2} w /\left(1+u^{2}\right) \in L^{\infty}\left(Q_{T}\right), g_{2}=u-v \in L^{\infty}\left(Q_{T}\right)$. It follows from Lemmas 4.1, 4.5, and (4.39) that $u, v, w, \nabla w$ are bounded. Thus applying Theorem 10.1 [37, Page 204] to (4.40) leads to

$$
u, v \in C^{\lambda_{3}, \lambda_{3} / 2}\left(\bar{Q}_{T}\right), \quad 0<\lambda_{3}<1 .
$$

We rewrite the third equation of (1.4) as

$$
w_{t}=\left(d_{3}+2 \alpha_{33} w\right) \Delta w+g_{3}(x, t)
$$

where $g_{3}=2 \alpha_{33}|\nabla w|^{2}-k w-\gamma w^{2}+\alpha u^{2} w /\left(1+u^{2}\right) \in C^{\lambda_{3}, \lambda_{3} / 2}\left(\bar{Q}_{T}\right)$. Applying Schauder estimate [29, Theorem 3.2.6, page 114] to (4.42) gives

$$
w \in C^{2+\lambda_{4},\left(2+\lambda_{4}\right) / 2}\left(\bar{Q}_{T}\right), \quad \text { where } \lambda_{4}=\min \left\{\lambda, \lambda_{3}\right\}
$$

Let

$$
u_{2}=\left(d_{1}+\alpha_{11} u+\alpha_{13} w\right) u, \quad v_{2}=\left(d_{2}+\alpha_{22} v\right) v,
$$

then

$$
\begin{gathered}
u_{2 t}=\left(d_{1}+2 \alpha_{11} u+\alpha_{13} w\right) \Delta u_{2}+g_{4}(x, t), \\
v_{2 t}=\left(d_{2}+2 \alpha_{22} v\right) \Delta v_{2}+g_{5}(x, t),
\end{gathered}
$$


where $g_{4}=\left(d_{1}+2 \alpha_{11} u+\alpha_{13} w\right)\left(\beta v-a u-b u^{2}-c u^{3}-u^{2} w /\left(1+u^{2}\right)\right)+\alpha_{13} u w_{t}, g_{5}=\left(d_{2}+2 \alpha_{22} v\right)(u-v)$. From (4.41), we have $d_{1}+2 \alpha_{11} u+\alpha_{13} w, d_{2}+2 \alpha_{22} v \in C^{\lambda_{3}, \lambda_{3} / 2}\left(\bar{Q}_{T}\right)$. It follows from (4.41) and (4.43) that $g_{4}(x, t), g_{5}(x, t) \in C^{\lambda_{4}, \lambda_{4} / 2}\left(\bar{Q}_{T}\right)$. Applying Schauder estimate to (4.45) gives

$$
u_{2}, v_{2} \in C^{2+\lambda_{4},\left(2+\lambda_{4}\right) / 2}\left(\bar{Q}_{T}\right)
$$

Solving equations (4.44) for $u, v$, respectively, we have

$$
u, v \in C^{2+\lambda_{4},\left(2+\lambda_{4}\right) / 2}\left(\bar{Q}_{T}\right)
$$

In particular, to conclude $u, v, w \in C^{2+\lambda,(2+\lambda) / 2}\left(\bar{Q}_{T}\right)$, we need to repeat the above bootstrap technique. Since $T$ is arbitrary, so the classical solution $(u, v, w)$ of $(1.4)$ exists globally in time.

Now we discuss the global stability of the positive equilibrium $E^{*}\left(u^{*}, v^{*}, w^{*}\right)$ (see Section 2) for (1.4).

Theorem 4.8. Assume that the all conditions in Theorem 4.2, (2.1), and

$$
\frac{1}{\beta}\left(a+b u^{*}+c u^{* 2}\right)>2+\frac{\left(u^{*}+\sqrt{1+u^{* 2}}\right)^{2}}{8}+\frac{u^{* 4}}{2 \beta^{2}}, \quad \frac{\gamma}{\alpha}>\frac{1}{2\left(1+u^{* 2}\right)^{2}}
$$

hold. Let $\left(u^{*}, v^{*}, w^{*}\right)$ be the unique positive equilibrium point of $(1.4)$, and let $(u, v, w)$ be a positive solution for (1.4). Then

$$
\left\|u(\cdot, t)-u^{*}\right\|_{L^{2}(\Omega)} \longrightarrow 0, \quad\left\|v(\cdot, t)-v^{*}\right\|_{L^{2}(\Omega)} \longrightarrow 0, \quad\left\|w(\cdot, t)-w^{*}\right\|_{L^{2}(\Omega)} \longrightarrow 0 \quad(t \longrightarrow \infty)
$$

provided that $d_{1} \cdot d_{2} \cdot d_{3}$ is large enough.

Proof. Define the Lyapunov function

$$
H(u, v, w)=\frac{1}{2 \beta} \int_{\Omega}\left(u-u^{*}\right)^{2} d x+\frac{1}{2} \int_{\Omega}\left(v-v^{*}\right)^{2} d x+\frac{1}{\alpha} \int_{\Omega}\left(w-w^{*}-w^{*} \ln \frac{w}{w^{*}}\right) d x .
$$


Let $(u, v, w)$ be a positive solution of (1.4), Then

$$
\begin{aligned}
\frac{d H}{d t} \leq & -\int_{\Omega}\left[\frac{1}{\beta}\left(d_{1}+2 \alpha_{11} u+\alpha_{13} w\right)|\nabla u|^{2}+\left(d_{2}+2 \alpha_{22} v\right)|\nabla v|^{2}\right. \\
& \left.+\frac{1}{\alpha}\left(d_{3}+2 \alpha_{33} w\right) \frac{w^{*}}{w^{2}}|\nabla w|^{2}+\frac{1}{\beta} \alpha_{13} u \nabla u \nabla w\right] d x \\
& -\int_{\Omega}\left\{\left(u-u^{*}\right)^{2} \frac{1}{\beta}\left[a+b\left(u+u^{*}\right)+c\left(u^{2}+u u^{*}+u^{* 2}\right)+\frac{w\left(u+u^{*}\right)}{\left(1+u^{2}\right)\left(1+u^{* 2}\right)}\right]-2\right. \\
& \left.-\frac{1}{2}\left(\frac{u+u^{*}}{1+u^{2}}-\frac{u^{* 2}}{\beta}\right)^{2}\right\} d x-\frac{1}{2} \int_{\Omega}\left(v-v^{*}\right)^{2} d x \\
& -\int_{\Omega}\left(w-w^{*}\right)^{2}\left[\frac{r}{\alpha}-\frac{1}{2\left(1+u^{* 2}\right)^{2}}\right] d x
\end{aligned}
$$

The first integrand in the right hand of the previous inequality is positive definite if

$$
\frac{4 \beta}{\alpha} w^{*}\left(d_{1}+2 \alpha_{11} u+\alpha_{13} w\right)\left(d_{2}+2 \alpha_{22} v\right)\left(d_{3}+2 \alpha_{33} w\right)>\alpha_{13}^{2} u^{2} w^{2}\left(d_{2}+2 \alpha_{22} v\right) .
$$

Therefore, when the all conditions in Theorem 4.8 hold, there exists a positive constant $\delta$ such that

$$
\frac{d H(u, v, w)}{d t} \leq-\delta \int_{\Omega}\left[\left(u-u^{*}\right)^{2}+\left(v-v^{*}\right)^{2}+\left(w-w^{*}\right)^{2}\right] d x
$$

This implies that $\left\|u(\cdot, t)-u^{*}\right\|_{L^{2}(\Omega)},\left\|v(\cdot, t)-v^{*}\right\|_{L^{2}(\Omega)},\left\|w(\cdot, t)-w^{*}\right\|_{L^{2}(\Omega)} \rightarrow 0$ as $t \rightarrow \infty$. So the proof of Theorem 4.8 is completed.

\section{Acknowledgments}

This work has been partially supported by the China National Natural Science Foundation (no. 10871160), the NSF of Gansu Province (no. 096RJZA118), the Scientific Research Fund of Gansu Provincial Education Department, and NWNU-KJCXGC-03-47 Foundation.

\section{References}

[1] W. G. Aiello and H. I. Freedman, "A time-delay model of single-species growth with stage structure," Mathematical Biosciences, vol. 101, no. 2, pp. 139-153, 1990.

[2] X. Zhang, L. Chen, and A. U. Neumann, "The stage-structured predator-prey model and optimal harvesting policy," Mathematical Biosciences, vol. 168, no. 2, pp. 201-210, 2000.

[3] S. Liu, L. Chen, and Z. Liu, "Extinction and permanence in nonautonomous competitive system with stage structure," Journal of Mathematical Analysis and Applications, vol. 274, no. 2, pp. 667-684, 2002.

[4] Z. Lin, "Time delayed parabolic system in a two-species competitive model with stage structure," Journal of Mathematical Analysis and Applications, vol. 315, no. 1, pp. 202-215, 2006. 
[5] R. Xu, "A reaction-diffusion predator-prey model with stage structure and nonlocal delay," Applied Mathematics and Computation, vol. 175, no. 2, pp. 984-1006, 2006.

[6] R. Xu, M. A. J. Chaplain, and F. A. Davidson, "Global convergence of a reaction-diffusion predatorprey model with stage structure for the predator," Applied Mathematics and Computation, vol. 176, no. 1, pp. 388-401, 2006.

[7] R. Xu, M. A. J. Chaplain, and F. A. Davidson, "Global convergence of a reaction-diffusion predatorprey model with stage structure and nonlocal delays," Computers $\mathcal{E}$ Mathematics with Applications, vol. 53, no. 5, pp. 770-788, 2007.

[8] M. Wang, "Stability and Hopf bifurcation for a prey-predator model with prey-stage structure and diffusion," Mathematical Biosciences, vol. 212, no. 2, pp. 149-160, 2008.

[9] Z. Wang and J. Wu, "Qualitative analysis for a ratio-dependent predator-prey model with stage structure and diffusion," Nonlinear Analysis: Real World Applications, vol. 9, no. 5, pp. 2270-2287, 2008.

[10] G. Galiano, M. L. Garzón, and A. Jüngel, "Semi-discretization in time and numerical convergence of solutions of a nonlinear cross-diffusion population model," Numerische Mathematik, vol. 93, no. 4, pp. 655-673, 2003.

[11] L. Chen, Mathematical Models and Methods in Ecology, Science Press, Beijing, China, 1988.

[12] L. J. Chen and J. H. Sun, "The uniqueness of a limit cycle for a class of Holling models with functional responses," Acta Mathematica Sinica, vol. 45, no. 2, pp. 383-388, 2002.

[13] W.-T. Li and S.-L. Wu, "Traveling waves in a diffusive predator-prey model with Holling type-III functional response," Chaos, Solitons E Fractals, vol. 37, no. 2, pp. 476-486, 2008.

[14] W. Ko and K. Ryu, "Qualitative analysis of a predator-prey model with Holling type II functional response incorporating a prey refuge," Journal of Differential Equations, vol. 231, no. 2, pp. 534-550, 2006.

[15] H. Zhang, P. Georgescu, and L. Chen, "An impulsive predator-prey system with BeddingtonDeAngelis functional response and time delay," International Journal of Biomathematics, vol. 1, no. 1, pp. 1-17, 2008.

[16] Y. Fan, L. Wang, and M. Wang, "Notes on multiple bifurcations in a delayed predator-prey model with nonmonotonic functional response," International Journal of Biomathematics, vol. 2, no. 2, pp. 129-138, 2009.

[17] F. Wang and Y. An, "Existence of nontrivial solution for a nonlocal elliptic equation with nonlinear boundary condition," Boundary Value Problems, vol. 2009, Article ID 540360, 8 pages, 2009.

[18] Y. Lou and W.-M. Ni, "Diffusion, self-diffusion and cross-diffusion," Journal of Differential Equations, vol. 131, no. 1, pp. 79-131, 1996.

[19] Y. Lou, W.-M. Ni, and Y. Wu, "On the global existence of a cross-diffusion system," Discrete and Continuous Dynamical Systems, vol. 4, no. 2, pp. 193-203, 1998.

[20] S.-A. Shim, "Uniform boundedness and convergence of solutions to cross-diffusion systems," Journal of Differential Equations, vol. 185, no. 1, pp. 281-305, 2002.

[21] S.-A. Shim, "Uniform boundedness and convergence of solutions to the systems with cross-diffusions dominated by self-diffusions," Nonlinear Analysis: Real World Applications, vol. 4, no. 1, pp. 65-86, 2003.

[22] Y. S. Choi, R. Lui, and Y. Yamada, "Existence of global solutions for the Shigesada-Kawasaki-Teramoto model with weak cross-diffusion," Discrete and Continuous Dynamical Systems, vol. 9, no. 5, pp. 1193 1200, 2003.

[23] Y.S. Choi, R. Lui, and Y. Yamada, "Existence of global solutions for the Shigesada-Kawasaki-Teramoto model with strongly coupled cross-diffusion," Discrete and Continuous Dynamical Systems, vol. 10, no. 3, pp. 719-730, 2004.

[24] P. Y. H. Pang and M. X. Wang, "Existence of global solutions for a three-species predator-prey model with cross-diffusion," Mathematische Nachrichten, vol. 281, no. 4, pp. 555-560, 2008.

[25] S. Fu, Z. Wen, and S. Cui, "Uniform boundedness and stability of global solutions in a strongly coupled three-species cooperating model," Nonlinear Analysis: Real World Applications, vol. 9, no. 2, pp. 272-289, 2008.

[26] F. Yang and S. Fu, "Global solutions for a tritrophic food chain model with diffusion," The Rocky Mountain Journal of Mathematics, vol. 38, no. 5, pp. 1785-1812, 2008.

[27] B. Dubey, B. Das, and J. Hussain, "A predator-prey interaction model with self and cross-diffusion," Ecological Modelling, vol. 141, no. 1-3, pp. 67-76, 2001.

[28] J. K. Hale, Ordinary Differential Equations, Krieger, Malabar, Fla, USA, 1980.

[29] Q. Ye and Z. Li, Introduction to Reaction-Diffusion Equations, Science Press, Beijing, China, 1999. 
[30] D. Henry, Geometric Theory of Semilinear Parabolic Equations, vol. 840 of Lecture Notes in Mathematics, Springer, Berlin, Germany, 1993.

[31] Z. Lin and M. Pedersen, "Stability in a diffusive food-chain model with Michaelis-Menten functional response," Nonlinear Analysis: Theory, Methods \& Applications, vol. 57, no. 3, pp. 421-433, 2004.

[32] M. Wang, Nonliear Parabolic Equation of Parabolic Type, Science Press, Beijing, China, 1993.

[33] K. J. Brown, P. C. Dunne, and R. A. Gardner, "A semilinear parabolic system arising in the theory of superconductivity," Journal of Differential Equations, vol. 40, no. 2, pp. 232-252, 1981.

[34] H. Amann, "Dynamic theory of quasilinear parabolic equations. I. Abstract evolution equations," Nonlinear Analysis: Theory, Methods E Applications, vol. 12, no. 9, pp. 895-919, 1988.

[35] H. Amann, "Dynamic theory of quasilinear parabolic equations. II. Reaction-diffusion systems," Differential and Integral Equations, vol. 3, no. 1, pp. 13-75, 1990.

[36] H. Amann, "Dynamic theory of quasilinear parabolic systems. III. Global existence," Mathematische Zeitschrift, vol. 202, no. 2, pp. 219-250, 1989.

[37] O. A. Ladyženskaja, V. A. Solonnikov, and N. N. Ural'ceva, Linear and Quasilinear Equations of Parabolic Type, vol. 23 of Translations of Mathematical Monographs, American Mathematical Society, Providence, RI, USA, 1967. 